Review began 02/23/2022 Review ended 02/25/2022 Published 03/01/2022

๑) Copyright 2022

Brgdar et al. This is an open access article distributed under the terms of the Creative Commons Attribution License CC-BY 4.0. which permits unrestricted use, distribution, and reproduction in any medium, provided the original author and source are credited.

\section{Effects of Substance Use Disorder on In-Hospital Outcomes of Young Patients Presenting With a Cardiovascular Event: A Nationwide Analysis}

Ahmed Brgdar ${ }^{1}$, John Gharbin ${ }^{1}$, Ayman Elawad ${ }^{1}$, Jin Yi ${ }^{1}$, Jacob Sanchez ${ }^{1}$, Adey Bishaw ${ }^{1}$, Mohamed E. Taha $^{2}$, Edmund Essah Ameyaw ${ }^{3}$, Norman Allen ${ }^{4}$, Mehrotra Prafulla ${ }^{2}$

1. Internal Medicine, Howard University Hospital, Washington, DC, USA 2. Cardiovascular Medicine, Howard University Hospital, Washington, DC, USA 3. Department of Mathematics, Howard University College of Medicine, Washington, DC, USA 4. Cardiovascular Diseases, Providence Hospital, Washington, DC, USA

Corresponding author: Ahmed Brgdar, ahmedbrgdar@gmail.com

\section{Abstract \\ Background}

Substance use is widely prevalent among young adults and is associated with increased cardiovascular morbidity and mortality such as sudden cardiac arrest, acute coronary syndrome, arrhythmias, and cardiomyopathy. However, they are limited studies analyzing the impact of substance use disorder on inhospital outcomes among young patients with cardiovascular events.

\section{Methods}

All patients aged 18-39 years admitted primarily for major cardiovascular events including acute myocardial infarction (AMI), arrhythmia, cardiac arrest, acute ischemic stroke, and venous thromboembolic events in 2019 were identified in the National Inpatient Sample database. They were then categorized into those with and without concomitant substance use disorder (SUD). The primary outcome was in-hospital mortality. Unadjusted and adjusted analysis was performed on appropriate variables of interest.

\section{Results}

Of 57,985 hospitalizations with cardiac events, 12,115 (20\%) of young adults had concomitant SUD. SUD was significantly associated with cardiac arrest (OR 3.3; CI 2.4-4.4), atrial fibrillation (OR 1.5; CI 1.3-1.7), AMI (OR 1.3; CI 1.2-1.6), heart failure (OR 2.6; CI 2.4-3.0) (all p<0.05) despite a lower prevalence of traditional cardiovascular risk factors than non-users. Logistic regression showed acute kidney injury (aOR 1.5; CI 1.3$1.8 ; \mathrm{p}<0.001$ ) and inpatient mortality (aOR 1.6 ; CI $1.2-2.2$; $\mathrm{p}<0.001$ ) were also significantly higher in young patients presenting with cardiac events and concomitant SUD. There was no difference in the length of stay or incidence of gastrointestinal bleed between the two groups.

\section{Conclusion}

In young patients presenting with a cardiovascular event, concurrent substance use disorder was associated with increased in-hospital mortality despite significantly lower comorbidities.

Categories: Cardiology, Internal Medicine, Substance Use and Addiction

Keywords: alcohol, cannabis, young adults, substance use disorder, cardiac event

\section{Introduction}

Substance abuse has remained a persistent problem in the United States with increasing prevalence. Nearly 101, 263 drug overdose deaths were documented in the 12-month period ending in July 2021, increasing by $76.5 \%$ from 57,372 deaths during the same period five years previously [1]. Moreover, substance abuse is disproportionately higher among younger adults in the US. Young adults aged 18-25 years-old report a significantly higher past-month, past-year, and lifetime prevalence of prescription opioids, stimulants, and tranquilizers abuse than older age groups [2] and are the most likely of any age group to die from a prescription drug overdose [3]. Further, a recent analysis of the Veterans Health Administration data showed a $12.8 \%$ overall prevalence of substance use disorder (SUD) with a $27.7 \%$ prevalence in the group aged $18-34$ years old, which was the highest among any age group [4].

Although several studies have reported the cardiovascular outcomes associated with specific substances, many patients with SUD report polysubstance abuse [5,6]. Over $25 \%$ of patients with lifetime alcohol use disorder and over $80 \%$ of non-alcohol drug-specific SUD (cannabis, cocaine, heroin, hallucinogens, inhalants, prescription opioids, sedatives, tranquilizers, stimulants, or other drugs) report at least one other SUD [6]. As with the prevalence of SUD discussed above, young adults aged 18-29 years old had the highest odds of multiple SUDs [6]. However, there is a paucity of studies assessing the cardiovascular effects of 
cannabis, stimulants, alcohol, opioid, cocaine, psychoactive agents, hallucinogens, sedatives, hypnotics, and inhalants as a whole. In addition, little is known about the in-hospital outcomes of patients with SUD hospitalized primarily due to a cardiac event [7-9].

The impact of a combination of substances may be clinically more relevant than the effects of an individual substance. For instance, Rumalla et al. concluded that contaminant use of tobacco, cocaine, or amphetamines with cannabis was associated with increased odds of hospitalization for acute ischemic stroke while combining cannabis with opioid or alcohol was associated with lower odds [10]. Similarly, in the recently reported Veterans Affairs Healthcare database study, the risk of premature atherosclerotic cardiovascular diseases was the lowest among cocaine and cannabis users compared to the combinations of amphetamine with cocaine, cannabis, or both $[11,12]$.

Assessing the impact of SUD as a whole becomes even more imperative given that commonly abused substances often have contrasting effects on traditional CVD risk factors. A null association has been reported with cannabis use and central obesity, hyperlipidemia, diabetes, or hypertension [13]. On the other hand, methamphetamine and cocaine abuse had been negatively associated with obesity, hyperlipidemia, and hypertension $[9,14,15]$. Furthermore, although patients with opioid use disorder tend to have obesity and hyperlipidemia, most remain normotensive [16,17]. In contrast, alcohol increases the risk of cardiovascular diseases by direct action on the cardiovascular system as well as through traditional CVD risk factors, particularly obesity, hypertension, and dyslipidemia [18].

With the objective of bridging some of these gaps, in this study, we have investigated the impact of substance abuse on in-hospital outcomes among patients with cardiovascular events using hospitalization records from the 2019 National Inpatient Sample database (NIS).

\section{Materials And Methods}

Data source

We conducted a retrospective cohort study using the NIS database. NIS is the largest inpatient registry in the US with publicly available hospitalization records from 48 participating states and the District of Columbia [19].

\section{Study population}

The NIS database was queried using the International Classification of Diseases, Tenth Revision Clinical Modification (ICD-10-CM). Patients aged 18-39 years old admitted primarily for cardiac arrest, atrial fibrillation, and flutter, other cardiac arrhythmias, acute myocardial infarction, pulmonary embolism, heart failure, or acute ischemic stroke in 2019 were identified using the ICD-10-CM codes I46, I48, I49, I21, I26, I50, I63.9, respectively. These constituted cardiac events. From this data, a subset of patients with concomitant alcohol, cocaine, cannabis, hallucinogen, inhalant, opioid, psychoactive agents, stimulants, or sedatives related disorders were identified using the subcategory codes F10, F14, F12, F16, F18, F11, F19, F15, F13, respectively, and combined for substance use disorder. Substance use disorder (SUD) included substance abuse, dependence, use with intoxication, and substance use unspecified according to ICD-10-CM classification. Data were obtained on patient characteristics and hospital-level characteristics. Baseline patient characteristics included age, gender, race, hypertension, diabetes mellitus, obesity, chronic kidney disease, hyperlipidemia, hyperthyroidism. Hospital characteristics including teaching status. Although tobacco use has a strong independent association with adverse cardiovascular outcomes, we excluded patients with tobacco use disorder in line with previous studies [20]. Moreover, recent studies have shown exacerbated cardiovascular outcomes with substances like cannabis and cocaine in the absence of tobacco use compared to patients with concomitant tobacco use disorder [21,22].

\section{Outcomes}

The primary outcome of interest was in-hospital mortality. Secondary outcomes of interest were the length of stay, acute kidney injury (AKI), and gastrointestinal hemorrhage.

\section{Statistical analysis}

Data were analyzed with Software for Statistics and Data Science (STATA/SE 17.0, Stata Corp, Texas, US). Univariate analyses of cardiac events for the subpopulation were conducted. Multivariate logistic regression was used to adjust for confounders, e.g. age, gender, race, hypertension, diabetes mellitus, obesity, chronic kidney disease, hyperlipidemia, hyperthyroidism, hypothyroidism, nicotine dependence, and Charlson Comorbidity Index (CCI) [23], and hospital teaching status. Continuous variables were expressed as means $(95 \% \mathrm{CI})$ and used t-tests or regression to compare differences between exposure and non-exposure groups. Similarly, the chi-squared test was used to compare differences between categorical variables. A two-sided $\mathrm{p}<0.05$ was considered significant throughout the analyses.

\section{Results}




\section{Cureus}

A total of 57,985 patients aged 18-39 years old were admitted with cardiac events (including cardiac arrest, atrial fibrillation, and flutter, other cardiac arrhythmias, acute myocardial infarction, pulmonary embolism, heart failure, or acute ischemic stroke) in 2019, out of which 12,115 (20.89\%) had SUD. Patients with or without SUD were of similar age (32.7 years vs. 32.5 years), and over half of the patients in both groups were white (52.2\% vs. $52.4 \%$ ) (Table 1 ). Over $75 \%$ of the patients in both groups were treated at teaching hospitals (76.0\% vs. $77.0 \%$ ). Medicaid was the most common insurer for patients with SUD (50.8\%), while $48.5 \%$ of the patients without SUD were covered by private insurance HMO (Table 1). Among patients with SUD, cannabis was the most widely used substance (26\%).

\begin{tabular}{|c|c|c|c|}
\hline & SUD $(n=12,115)$ & No SUD $(n=45,870)$ & p-value \\
\hline Mean age, years & 32.7 (95\% Cl:32.6-33.0) & 32.5 (95\% Cl: 32.4-32.7) & 0.16 \\
\hline \multicolumn{4}{|l|}{$\operatorname{Sex}(\%)$} \\
\hline Female & $28.4 \%$ & $45.0 \%$ & \multirow{2}{*}{$<0.001$} \\
\hline Male & $71.6 \%$ & $55.0 \%$ & \\
\hline \multicolumn{4}{|l|}{ Race (\%) } \\
\hline White & $52.2 \%$ & $52.4 \%$ & \multirow{6}{*}{$<0.001$} \\
\hline Black & $27.0 \%$ & $28.2 \%$ & \\
\hline Hispanic & $13.7 \%$ & $12.0 \%$ & \\
\hline Asian/Pacific Islander & $2.2 \%$ & $3.0 \%$ & \\
\hline Native American & $2.0 \%$ & $0.7 \%$ & \\
\hline Other & $2.8 \%$ & $3.7 \%$ & \\
\hline \multicolumn{4}{|l|}{ Hospital Characteristics (\%) } \\
\hline Teaching hospital & $76.0 \%$ & $77.0 \%$ & \multirow{2}{*}{0.311} \\
\hline Non-teaching hospital & $24.0 \%$ & $23.0 \%$ & \\
\hline \multicolumn{4}{|l|}{ Type of Insurance (\%) } \\
\hline Medicare & $6.0 \%$ & $8.6 \%$ & \multirow{4}{*}{$<0.001$} \\
\hline Medicaid & $50.8 \%$ & $31.0 \%$ & \\
\hline Private insurance/ $\mathrm{HMO}^{\ddagger}$ & $23.5 \%$ & $48.5 \%$ & \\
\hline Self-Pay & $19.8 \%$ & $11.9 \%$ & \\
\hline \multicolumn{4}{|l|}{ Type of Substance Use (\%) } \\
\hline Cannabis & $26.0 \%$ & - & - \\
\hline Stimulants & $23.4 \%$ & - & - \\
\hline Alcohol & $20.8 \%$ & - & - \\
\hline Opioid & $10.9 \%$ & - & - \\
\hline Cocaine & $9.3 \%$ & - & - \\
\hline Psychoactive Agents & $9.0 \%$ & - & - \\
\hline Hallucinogens & $0.3 \%$ & - & - \\
\hline Sedatives/hypnotics & $0.2 \%$ & - & - \\
\hline Inhalant & $0.0 \%$ & - & - \\
\hline \multicolumn{4}{|l|}{ Comorbidities (\%) } \\
\hline Hypertension & $19.7 \%$ & $23.3 \%$ & 0.003 \\
\hline Diabetes mellitus & $1.7 \%$ & $2.3 \%$ & 0.084 \\
\hline Obesity & $23.8 \%$ & $34.9 \%$ & $<0.001$ \\
\hline
\end{tabular}




\section{Cureus}

\begin{tabular}{|llll|}
\hline Chronic kidney disease & $5.8 \%$ & $7.8 \%$ & 0.002 \\
\hline Hyperlipidemia & $14.0 \%$ & $20.3 \%$ & $<0.001$ \\
\hline Hyperthyroidism & $0.9 \%$ & $1.3 \%$ & 0.186 \\
\hline Hypothyroidism & $2.9 \%$ & $5.3 \%$ & $<0.001$ \\
\hline Mean CCI & $1.45(95 \%$ Cl:1.39-1.50) & $1.41(95 \%$ Cl: $1.38-1.45)$ & 0.281 \\
\hline
\end{tabular}

TABLE 1: Baseline demographics and comorbidities of 57,985 young adults aged 18-39 years old admitted with cardiac events with and without substance use disorder (SUD).

‡HMO = Health maintenance organization; ${ }^{*} \mathrm{CCl}=$ Charlson Comorbidity Index; \#LoS = Length of hospital stay in days; §GI bleed = gastrointestinal hemorrhage

A greater proportion of patients with SUD were male (71.6\% vs. 55.0\%). Patients with SUD also had significantly lower prevalence of hypertension (19.7\% vs. $23.3 \%$, p=0.003), obesity ( $23.8 \%$ vs. $34.9 \%$, $\mathrm{p}<0.001$ ), chronic kidney disease ( $5.8 \%$ vs. $7.8 \%$, $\mathrm{p}=0.002)$, hyperlipidemia ( $14.0 \%$ vs. $20.3 \%$, $\mathrm{p}<0.001)$, and hypothyroidism $(2.9 \%$ vs. $5.3 \%$, p $<0.001)$ although the mean CCI were similar between patients with or without SUD (1.45 vs. 1.41, $\mathrm{p}=0.281$ ) (Table 1). The prevalence of diabetes mellitus was numerically lower in the SUD group (1.7\% vs. $2.3 \%, \mathrm{p}=0.084)$.

In the subpopulation univariate analysis, SUD was significantly associated with cardiac arrest (OR $=3.3(95 \%$ CI: 2.4-4.4), $\mathrm{p}<0.001)$, atrial fibrillation ( $\mathrm{OR}=1.5$ (95\% CI: 1.3-1.7), $\mathrm{p}<0.001)$, myocardial infarction $(\mathrm{OR}=1.3$ (95\% CI 1.2-1.6), p<0.001), pulmonary embolism (OR = 1.0 (95\% CI: 0.8-1.0), p=0.020), and heart failure (OR $=2.6$ (95\% CI: $2.4-3.0), \mathrm{p}<0.001)$ but not of other unspecified arrhythmias (OR $=1.2$ (95\% CI: 1.0-1.5), $\mathrm{p}=0.074)$ or acute ischemic stroke $(\mathrm{OR}=1.1$ (95\% CI: 0.9-1.4), $\mathrm{p}=0.261)$ (Table 2).

\begin{tabular}{|c|c|c|}
\hline Cardiac Events & Odds Ratio $(95 \% \mathrm{Cl})$ & p-value \\
\hline Cardiac arrest & $3.3(2.4-4.4)$ & $<0.001$ \\
\hline Atrial fibrillation and flutter & $1.5(1.3-1.7)$ & $<0.001$ \\
\hline Arrhythmias (atrial fibrillation and atrial flutter excluded) & $1.2(1.0-1.5)$ & 0.074 \\
\hline Myocardial infarction & $1.3(1.2-1.6)$ & $<0.001$ \\
\hline Pulmonary embolism & $1.0(0.8-1.0)$ & 0.020 \\
\hline Heart failure & $2.6(2.4-3.0)$ & $<0.001$ \\
\hline Acute ischemic stroke & $1.1(0.9-1.4)$ & 0.261 \\
\hline
\end{tabular}

TABLE 2: Sub-population univariate analysis of cardiac events stratified by substance use disorder

Furthermore, patients presenting with cardiac events with concomitant SUD had a significantly greater risk of in-hospital mortality ( $\mathrm{OR}=1.5$ (95\% CI: $1.2-2.0)$, $\mathrm{p}<0.001)$, and acute kidney failure ( $\mathrm{OR}=1.6$ (95\% CI: $1.4-1.8), \mathrm{p}<0.001)$. However SUD did not have statistical significant association with gastrointestinal hemorrhage $(\mathrm{OR}=1.4$ (95\% CI: 0.6-2.9), $\mathrm{p}=0.421$ ) or the length of hospitalization (OR $=0.9$ (95\% CI: 0.7-1.1), $\mathrm{p}=0.383$ ) (Table 3). The risk of in-hospital mortality and acute kidney failure remained elevated even after adjusting for potential confounders in the multivariate analysis (Table 3). 


\section{Cureus}

\begin{tabular}{|c|c|c|c|c|}
\hline \multirow{2}{*}{ Outcomes } & \multicolumn{2}{|l|}{ Univariate } & \multicolumn{2}{|l|}{ Multivariate } \\
\hline & Odds Ratio (95\% Cl) & $\mathrm{p}$-value & Adjusted Odds Ratio (95\% Cl) & $\mathrm{p}$-value \\
\hline In-hospital mortality & $1.5(1.2-2.0)$ & $<0.001$ & $1.6(1.2-2.2)$ & 0.001 \\
\hline Acute kidney failure & $1.6(1.4-1.8)$ & $<0.001$ & $1.5(1.3-1.8)$ & $<0.001$ \\
\hline GI bleed§ & $1.4(0.6-2.9)$ & 0.421 & $1.2(0.5-2.7)$ & 0.584 \\
\hline Length of stay in days & $0.9(0.7-1.1)$ & 0.383 & $0.9(0.7-1.1)$ & 0.452 \\
\hline \multicolumn{5}{|c|}{$\begin{array}{l}\text { TABLE 3: Logistic regression analysis of in-hospital outcomes of } 57,985 \text { young adults aged } 18-3 \\
\text { years old admitted with cardiac events with and without substance use disorder }\end{array}$} \\
\hline \multicolumn{5}{|c|}{$\S G I$ bleed = gastrointestinal hemorrhage } \\
\hline
\end{tabular}

\section{Discussion}

A growing body of literature suggests that young patients with SUD have exacerbated risk of CVD. A recently published analysis of the nationwide Veterans Affairs Healthcare database showed that young adults with premature atherosclerotic cardiovascular diseases reported significantly higher use of tobacco, alcohol, cocaine, amphetamine, and cannabis, all independently associated with premature atherosclerotic cardiovascular diseases [11]. The same study noted an increased risk of premature atherosclerotic cardiovascular diseases with each additional substance use, and the abuse of $\geqslant 4$ recreational substances was associated with a nine-fold increase in risk [11]. Our findings expand the scope of these earlier observations by demonstrating that SUD was significantly associated with cardiovascular outcomes, including cardiac arrest, atrial fibrillation, myocardial infarction, pulmonary embolism, and heart failure. Additionally, we observed higher in-hospital mortality and acute kidney failure rates among patients presenting with a cardiovascular event and concomitant SUD.

Our study showed an increased risk of adverse cardiovascular and in-hospital mortality outcomes among SUD patients despite a lower prevalence of traditional CVD risk factors, including hypertension, obesity, and hyperlipidemia. These findings are consistent with previous studies, although earlier studies have focused on single substances, which suggested that commonly abused drugs may produce adverse cardiovascular and mortality outcomes independent of traditional CVD risk factors [13,24,25]. For instance, methamphetamine has been shown to induce cardiomyopathy [26] despite a negative association between methamphetamine abuse and traditional CVD risk factors $[14,15]$. Also, a significantly higher proportion of cocaine users in the National Cardiovascular Data Registry ACTION Registry-GWTG database had myocardial infarction and cardiogenic shock at presentation despite the lower occurrence of traditional cardiovascular risk factors including obesity, diabetes, dyslipidemia, and hypertension [9]. Cocaine abuse is also known to induce cardiomyopathy, coronary artery spasm, and platelet activation resulting in an increased risk of coronary artery occlusion, myocardial infarction, sudden cardiovascular death, and all-cause mortality, especially in younger patients $[27,28]$.

In contrast, an exacerbated risk of cardiovascular events due to alcohol may be mediated by direct cardiovascular effects of the substances and through traditional CVD risk factors. Alcohol use disorder has been positively associated with several risk factors of CVD, particularly central obesity, hypertension, and hyperlipidemia [16,17,29,30], and has been implicated in the development of atrial fibrillation, nonischemic dilated cardiomyopathy, ischemic heart disease, and stroke with a dose-dependent increase in the risk of cardiovascular and all-cause mortality [31-33].

Opioid abuse may also increase the risk of cardiovascular events through direct action on traditional CVD risk factors, particularly obesity and hyperlipidemia [16,17]. Among patients with opioid overdose-related hospitalizations in the NIS database, $8.6 \%$ had at least one cardiovascular event including atrial fibrillation, myocardial infarction, or heart failure episode. There was a significantly higher cost of hospitalization, length of stay, and in-hospital mortality associated with opioid abuse and subsequent cardiovascular events [8]. A recent scoping review that explored the effects of chronic opioid use on CVD outcomes found a positive correlation between chronic opioid use and myocardial infarction [34]. However, other CVD outcomes, such as CAD, arrhythmia, stroke, and heart failure with prescription or non-prescription opioid abuse remain poorly studied [34].

Any potential association between SUD and traditional CVD risk factors or lack thereof must be interpreted along with the strength and limitations of diagnostic criteria used. For example, substance use disorders, specifically alcohol and opioid use are known to increase the constituent risk factors of metabolic syndrome $[35,36]$. However, several studies indicate that the association between SUD and metabolic syndrome 
depends on the diagnostic criteria used to define metabolic syndrome with International Diabetes Federation (IDF) criteria, generally associated with positive association, but not National Cholesterol Education Programme Adult Treatment Panel (NCEP ATP-III) criteria [17,36].

\section{Study limitations}

Our analysis did not distinguish between abuse of prescription and non-prescription drugs, and we were unable to examine additional confounders such as dose and duration of substance use due to limitations of the NIS database. Moreover, patients' socioeconomic status, which has been associated with the risk of both SUD $[37,38]$ and CVD $[39,40]$ among young adults subpopulations in the US, is another potential confounder not considered in our analysis. While other unmeasured confounders may exist, they are expected to be the same across all groups. Our analysis is limited to in-hospital outcomes of young adults aged 18-39 years and therefore may not generalize to long-term outcomes observed, for example, in older adults or adolescents. Also, the findings of this retrospective observational study need to be confirmed in future randomized control trials. Finally, there is an inherent risk of selection bias in any large database study due to coding errors or missing data, although the National Inpatient Sample auditing process is well established, minimizing data inaccuracy issues.

\section{Conclusions}

In young patients presenting with a cardiovascular event, concurrent substance use disorder was associated with increased in-hospital mortality and acute kidney injury risks despite significantly lower comorbidities. However, no statistically significant difference was observed in length of stay or incidence of gastrointestinal hemorrhage between the two groups. Increasing community collaboration, controlling polysubstance use, and raising awareness of the damaging effects of substance use, especially among young adults is needed.

\section{Additional Information \\ Disclosures}

Human subjects: Consent was obtained or waived by all participants in this study. Animal subjects: All authors have confirmed that this study did not involve animal subjects or tissue. Conflicts of interest: In compliance with the ICMJE uniform disclosure form, all authors declare the following: Payment/services info: All authors have declared that no financial support was received from any organization for the submitted work. Financial relationships: All authors have declared that they have no financial relationships at present or within the previous three years with any organizations that might have an interest in the submitted work. Other relationships: All authors have declared that there are no other relationships or activities that could appear to have influenced the submitted work.

\section{Acknowledgements}

The authors also acknowledge the contributions of Dr. Daniel Larbi of Howard University Hospital, DC, US, for his critical review of this manuscript.

\section{References}

1. National Center for Health Statistics. Provisional drug overdose death counts . (2022). Accessed: March 1, 2022: http://www.cdc.gov/nchs/nvss/vsrr/drug-overdose-data.htm.

2. Tapscott BE, Schepis TS: Nonmedical use of prescription medications in young adults . Adolesc Med State Art Rev. 2013, 24:597-610. 10.1542/9781581108736-nonmedical_use

3. National Institute on Drug Abuse. Abuse of prescription (Rx) drugs affects young adults most . (2016). Accessed: 04 February 2022: https://archives.drugabuse.gov/trends-statistics/abuse-prescription-rx-drugsaffects-young-adults-most.

4. Hoggatt KJ, Harris AH, Washington DL, Williams EC: Prevalence of substance use and substance-related disorders among US Veterans Health Administration patients. Drug Alcohol Depend. 2021, 225:108791. 10.1016/j.drugalcdep.2021.108791

5. Bailey AJ, Farmer EJ, Finn PR: Patterns of polysubstance use and simultaneous co-use in high risk young adults. Drug Alcohol Depend. 2019, 205:107656. 10.1016/j.drugalcdep.2019.107656

6. McCabe SE, West BT, Jutkiewicz EM, Boyd CJ: Multiple DSM-5 substance use disorders: A national study of US adults. Hum Psychopharmacol. 2017, 32: 10.1002/hup.2625

7. Desai R, Shamim S, Patel K, et al.: Primary causes of hospitalizations and procedures, predictors of inhospital mortality, and trends in cardiovascular and cerebrovascular events among recreational marijuana users: a five-year nationwide inpatient assessment in the United States. Cureus. 2018, 10:e3195. 10.7759/cureus.3195

8. Doshi R, Majmundar M, Kansara T, Desai R, Shah J, Kumar A, Patel K: Frequency of cardiovascular events and in-hospital mortality with opioid overdose hospitalizations. Am J Cardiol. 2019, 124:1528-33. 10.1016/j.amjcard.2019.07.068

9. Gupta N, Washam JB, Mountantonakis SE, Li S, Roe MT, de Lemos JA, Arora R: Characteristics, management, and outcomes of cocaine-positive patients with acute coronary syndrome (from the National Cardiovascular Data Registry). Am J Cardiol. 2014, 113:749-56. 10.1016/j.amjcard.2013.11.023

10. Rumalla K, Reddy AY, Mittal MK: Recreational marijuana use and acute ischemic stroke: A population-based 
analysis of hospitalized patients in the United States. J Neurol Sci. 2016, 364:191-6. 10.1016/j.jns.2016.01.066

11. Mahtta D, Ramsey D, Krittanawong C, et al.: Recreational substance use among patients with premature atherosclerotic cardiovascular disease. Heart. 2021, 107:650-6. 10.1136/heartjnl-2020-318119

12. Scott ML, Murnane KS, Orr AW: Young at heart? Drugs of abuse cause early-onset cardiovascular disease in the young. Heart. 2021, 107:604-6. 10.1136/heartjnl-2020-318856

13. Vidot DC, Prado G, Hlaing WM, Florez HJ, Arheart KL, Messiah SE: Metabolic syndrome among marijuana users in the united states: an analysis of national health and nutrition examination survey data. Am J Med. 2016, 129:173-9. 10.1016/j.amjmed.2015.10.019

14. Zhang M, Lv D, Zhou W, et al.: The levels of triglyceride and total cholesterol in methamphetamine dependence. Medicine (Baltimore). 2017, 96:e6631. 10.1097/MD.0000000000006631

15. Lv D, Zhang M, Jin X, et al.: The body mass index, blood pressure, and fasting blood glucose in patients with methamphetamine dependence. Medicine (Baltimore). 2016, 95:e3152. 10.1097/MD.0000000000003152

16. Bathla M, Singh M, Anjum S, Kulhara P, Jangli S IIrd: Metabolic syndrome in drug naïve patients with substance use disorder. Diabetes Metab Syndr. 2017, 11:167-71. 10.1016/j.dsx.2016.08.022

17. Singh Balhara YP, Jain R, Kuppili PP, Shukla A, Chawla N, Gupta R: Which criteria to use to identify metabolic syndrome among patients with addictive disorders?: observations among patients with alcohol and opioid dependence syndrome. Indian J Endocrinol Metab. 2018, 22:565-8. 10.4103/ijem.IJEM_617_17

18. Jarvis CM, Hayman LL, Braun LT, Schwertz DW, Ferrans CE, Piano MR: Cardiovascular risk factors and metabolic syndrome in alcohol- and nicotine-dependent men and women. J Cardiovasc Nurs. 2007, 22:42935. 10.1097/01.JCN.0000297387.21626.88

19. Agency for Healthcare Research and Quality. HCUP Nationwide Inpatient Sample (NIS). Healthcare Cost and Utilization Project (HCUP). (2021). Accessed: 18 November 2021: http://www.hcupus.ahrq.gov/nisoverview.jsp.

20. Lan CW, Fiellin DA, Barry DT, et al.: The epidemiology of substance use disorders in US Veterans: A systematic review and analysis of assessment methods. Am J Addict. 2016, 25:7-24. 10.1111/ajad.12319

21. Winhusen T, Theobald J, Kaelber DC, Lewis D: The association between regular cannabis use, with and without tobacco co-use, and adverse cardiovascular outcomes: cannabis may have a greater impact in nontobacco smokers. Am J Drug Alcohol Abuse. 2020, 46:454-61. 10.1080/00952990.2019.1676433

22. Winhusen T, Theobald J, Kaelber DC, Lewis D: The association between regular cocaine use, with and without tobacco co-use, and adverse cardiovascular and respiratory outcomes. Drug Alcohol Depend. 2020, 214:108136. 10.1016/j.drugalcdep.2020.108136

23. Charlson ME, Pompei P, Ales KL, MacKenzie CR: A new method of classifying prognostic comorbidity in longitudinal studies: development and validation. J Chronic Dis. 1987, 40:373-83. 10.1016/00219681(87)90171-8

24. Richards JR, Blohm E, Toles KA, Jarman AF, Ely DF, Elder JW: The association of cannabis use and cardiac dysrhythmias: a systematic review. Clin Toxicol (Phila). 2020, 58:861-9. 10.1080/15563650.2020.1743847

25. Auger N, Paradis G, Low N, Ayoub A, He S, Potter BJ: Cannabis use disorder and the future risk of cardiovascular disease in parous women: a longitudinal cohort study. BMC Med. 2020, 18:328. 10.1186/s12916-020-01804-6

26. Won S, Hong RA, Shohet RV, Seto TB, Parikh NI: Methamphetamine-associated cardiomyopathy. Clin Cardiol. 2013, 36:737-42. 10.1002/clc.22195

27. Rezkalla SH, Kloner RA: Cocaine-induced acute myocardial infarction. Clin Med Res. 2007, 5:172-6. 10.3121/cmr.2007.759

28. Kim ST, Park T: Acute and chronic effects of cocaine on cardiovascular health . Int J Mol Sci. 2019, 20:584. 10.3390/ijms20030584

29. Vancampfort D, Hallgren M, Mugisha J, De Hert M, Probst M, Monsieur D, Stubbs B: The prevalence of metabolic syndrome in alcohol use disorders: a systematic review and meta-analysis. Alcohol Alcohol. 2016, 51:515-21. 10.1093/alcalc/agw040

30. Puddey IB, Mori TA, Barden AE, Beilin LJ: Alcohol and hypertension-new insights and lingering controversies. Curr Hypertens Rep. 2019, 21:79. 10.1007/s11906-019-0984-1

31. Day E, Rudd JH: Alcohol use disorders and the heart. Addiction. 2019, 114:1670-8. 10.1111/add.14703

32. Roerecke M, Rehm J: Alcohol consumption, drinking patterns, and ischemic heart disease: a narrative review of meta-analyses and a systematic review and meta-analysis of the impact of heavy drinking occasions on risk for moderate drinkers. BMC Med. 2014, 12:182. 10.1186/s12916-014-0182-6

33. Ikehara S, Iso H, Yamagishi K, et al.: Alcohol consumption and risk of stroke and coronary heart disease among Japanese women: the Japan Public Health Center-based prospective study. Prev Med. 2013, 57:50510. 10.1016/j.ypmed.2013.07.003

34. Singleton JH, Abner EL, Akpunonu PD, Kucharska-Newton AM: Association of nonacute opioid use and cardiovascular diseases: a scoping review of the literature. J Am Heart Assoc. 2021, 10:e021260. 10.1161/JAHA.121.021260

35. Mattoo SK, Nebhinani N, Aggarwal M, Basu D, Kulhara P: Metabolic syndrome among substance dependent men: A study from north India. Ind Psychiatry J. 2013, 22:60-4. 10.4103/0972-6748.123631

36. Yousefzadeh G, Shokoohi M, Najafipour H, Eslami M, Salehi F: Association between opium use and metabolic syndrome among an urban population in Southern Iran: Results of the Kerman Coronary Artery Disease Risk Factor Study (KERCADRS). ARYA Atheroscler. 2015, 11:14-20.

37. Martin CC: High socioeconomic status predicts substance use and alcohol consumption in U.S. undergraduates. Subst Use Misuse. 2019, 54:1035-43. 10.1080/10826084.2018.1559193

38. Karriker-Jaffe KJ: Neighborhood socioeconomic status and substance use by U.S. adults . Drug Alcohol Depend. 2013, 133:212-21. 10.1016/j.drugalcdep.2013.04.033

39. Karlamangla AS, Singer BH, Williams DR, Schwartz JE, Matthews KA, Kiefe CI, Seeman TE: Impact of socioeconomic status on longitudinal accumulation of cardiovascular risk in young adults: the CARDIA Study (USA). Soc Sci Med. 2005, 60:999-1015. 10.1016/j.socscimed.2004.06.056

40. Zhang YB, Chen C, Pan XF, et al.: Associations of healthy lifestyle and socioeconomic status with mortality 


\section{Cureus}

and incident cardiovascular disease: two prospective cohort studies. BMJ. 2021, 373:n604. 10.1136/bmj.n604 